Proc. XIX International School of Semiconducting Compounds, Jaszowiec 1990

\title{
AUGER RECOMBINATION IN LOW-DIMENSIONAL PbSnTe STRUCTURES
}

\author{
M. MOCKER AND F. LEMKE \\ Humboldt University Berlin, Department of Physics, Invalidenstraße 110, Berlin 1040, \\ Germany \\ (Received August 8, 1990)
}

\begin{abstract}
IIow is the efficiency of Auger recombination in PbSnTe structures influenced by the reduction of dimensionality? Two points are investigated, the variation of matrix elements and of activation energies, respectively. It is shown that the latter one gives the more decisive effect. This effect is strongly anisotropic. Thus, $\{100\}$-planes or [100]-directions are fa voured to yield large Auger lifetimes for Q2D or Q1D structures, respectively.
\end{abstract}

PACS numbers: 72.10.-d

\section{Introduction}

Narrow gap semiconductors are interesting materials for infrared laser and detector applications. Due to the relatively large possible carrier populations near the band edge, low-dimensional structures promise improved diode properties such as decreased threshold current and weaker temperature dependence. A large amount of work has been done on III-V-QW lasers [1]. Semiconductor quantum well structures and superlattices on the base of lead chalcogenides have also been investigated experimentally and theoretically [2].

The clarification of the role of Auger recombination as a carrier lifetime limiting process is of particular importance since band-to-band Auger processes represent unavoidable non-radiative recombination channels. For bulk lead chalcogenides it is well-known [3] that increasing band structure anisotropy and decreasing gap energy (following e.g. the series $\mathrm{PbSSe}, \mathrm{PbSnSe}, \mathrm{PbSnTe}$ ) give rise to an increasing Auger recombination rate.

Here we ask, if the efficiency of Auger recombination is influenced by the reduction of dimensionality. Three essential points have to be considered in quasi-one-dimensional (Q1D) and quasi-two-dimensional (Q2D) structures: the occurence 
of new Auger recombination channels, the change in activation energy, $E_{\mathrm{a}}$, and the alteration of the relevant matrix elements.

For III-V low-dimensional structures it is generally found [4] that Auger recombination effects are as important in Q2D systems as in the bulk. However, Takeshima [5] has shown, that the directional dependence of the overlap integrals can reduce the effect remarkably in InGaAsP Q1D structures. The purpose of this paper is to investigate the problem theoretically for $\mathrm{PbTe} / \mathrm{Pb}_{1-x} \mathrm{Sn}_{x}$ Te quantum wells (Type I) in the rough approximation of infinite well depth.

\section{The activation energy}

A characteristic two-valley process [3] dominates the Auger recombination in lead chalcogenides with strong anisotropic band structure (e.g. $\mathrm{PbTe}, \mathrm{PbSnTe}$ ). The Auger process is possible only if the charge carriers in the initial states possess a minimal amount of energy, the activation energy, $E_{a}$. All $k$-vectors, $k_{i_{0}}$, of electron states involved in this transition have to be parallel or antiparallel, respectively. The activation energy for a certain direction of the $k_{i o}$ can be calculated by means of

$$
E_{\mathrm{a}}=\frac{E_{\mathrm{G}}}{2}\left[\sqrt{1+\frac{\sqrt{1+4 r}-1+2 r}{2(1-r)}}\left(2+\sqrt{\frac{2}{\sqrt{1+4 r}+1+2 r}}\right)-3\right] .
$$

Here, $r=r(\vartheta, \varphi)$ means a factor of anisotropy denoting the effective mass ratio $m_{\beta} / m_{\alpha}$ of the two valleys taking part in the process.

For Q1D structures we have to determine $r$ in the direction of free motion to get the activation energy by formula (1). For Q2D structures, there are certain directions in the plane of allowed $k$-vectors where $r$ is a minimum. This minimal $r$ will give the activation energy for the structure.

In bulk materials the problem will be solved by the absolute minimum of $r$ taken over all directions. For some specified directions of Q1D structures and planes of Q2D structures the results are given in the Table [6]. The most effective direction for the Auger process is the [111]-direction. In the [100]-direction with $r=1, E_{\mathrm{a}}=\infty$, we will get a vanishing Auger rate.

TABLE

Activation energies in low-dimensional $\mathrm{PbSnTe}$ structures.

\begin{tabular}{l|l|l|l|l|l}
\hline \multicolumn{2}{l|}{ Q2D } & \multicolumn{3}{l}{ Q1D } \\
\hline plane & $r$ & $E_{\mathrm{a}} / E_{\mathrm{G}}$ & direction & $r$ & $E_{\mathrm{a}} / E_{\mathrm{G}}$ \\
\hline$\{100\}$ & 0.4 & 0.54 & {$[100]$} & 1 & $\infty$ \\
& & & {$[110]$} & 0.4 & 0.54 \\
\hline$\{110\}$ & 0.1 & 0.1 & {$[100]$} & 1 & $\infty$ \\
& & & {$[110]$} & 0.4 & 0.54 \\
& & & {$[111]$} & 0.1 & 0.1 \\
\hline$\{111\}$ & 0.2 & 0.23 & {$[110]$} & 0.4 & 0.54 \\
& & & {$[211]$} & 0.2 & 0.23
\end{tabular}




\section{Calculation of Auger coefficients $C_{\mathrm{A}}$}

The complete analytical calculation of the Auger coefficients is possible only under strong approximations. We assume a two-band Kane-model with a Q2D subband dispersion relation at the $L$-point in $k$-space

$$
E_{n}\left(k_{x}, k_{y}\right)=-\frac{E_{\mathrm{G}}}{2} \pm \sqrt{\frac{E_{\mathrm{G}}^{2}}{4}+p_{x}^{2} k_{x}^{2}+p_{y}^{2} k_{y}^{2}+\frac{n^{2} \pi^{2}}{L^{2}} p_{z}^{2}}
$$

for an infinitely deep quantum well (well width $L$; momentum matrix elements $p_{x}, p_{y}, p_{z}$; bulk energy gap $E_{\mathrm{G}}$; upper and lower signs refer to conduction and valence band, respectively; subband index $n=1,2 \ldots$; the $x-y$-plane is the plane of free electron motion). Due to the Kramers degeneracy four basic states have to be considered at the band extrema. The main difficulties lie in the subsequent calculation of the matrix elements $M_{i f}$ between final states $f\left(1^{\prime}, 2^{\prime}\right)$ and initial states $i(1,2)$ and the summation over all states in

$$
C_{\mathrm{A}}^{2 \mathrm{D}}=\frac{1}{N_{1}^{2 \mathrm{D}} N_{1^{\prime}}^{2 \mathrm{D}} N_{2}^{2 \mathrm{D}}} \frac{2 \pi}{\hbar} \sum_{i f}\left|M_{i f}^{2 \mathrm{D}}\right|^{2} \delta\left(E_{i}-E_{f}\right) \exp \left[-\beta\left(E_{2^{\prime}}-E_{\mathrm{G}}\right)\right] .
$$

Within the Kane-model the effective density of states $N_{j}^{\nu \mathrm{D}}$ corresponding to the state $j$ (valley and band) takes the form

$$
N_{j}^{\nu \mathrm{D}}=\frac{\left(E_{\mathrm{G}} k_{\mathrm{B}} T\right)^{\frac{\nu}{2}}}{L^{3-\nu} \pi^{\frac{\nu}{2}} p^{\nu}} \frac{1}{2(\nu-1) !}\left[1+\frac{k_{\mathrm{B}} T}{E_{\mathrm{G}}} \frac{(\nu+2) ! !}{4}\left(1+(\nu-2) \frac{(\nu+4)}{8} \frac{k_{\mathrm{B}} T}{E_{\mathrm{G}}} \ldots\right)\right]
$$

where $\nu=1,2,3$ stands for Q1D, Q2D and 3D, respectively. For parabolic bands the expression in curly brackets equals one. $p^{\nu}$ denotes a product of momentum matrix elements which apply to the given direction, plane or bulk, respectively. $L^{3-\nu}$ is the length or the area of the confinement region.

Further we use a many-band envelope function approach [7] expanding the carrier wave function according to

$$
\Psi_{\nu K}^{n}(r)=\frac{1}{\sqrt{\Omega}} \exp (\mathrm{i} K R) \sum_{\nu^{\prime}} \chi_{\nu \nu^{\prime}}^{n}(z, K) u_{\nu^{\prime} o}(r) .
$$

Here the label $\nu^{\prime}$ runs over four $L$-point basic states. The envelope functions $\chi_{\nu \nu^{\prime}}^{n}$ depend on the in-plane wave vector $K$, the Bloch factors $u_{\nu^{\prime} o}$ are taken at the band extrema. The influence of remote bands we will neglect. To evaluate the matrix element $M_{i f}^{2 D}$ for the Auger transition we use the Fourier representation of the Coulomb potential and get

$$
M_{i f}^{2 \mathrm{D}}=\frac{1}{2 \pi} \frac{e^{2}}{A \varepsilon_{o} \varepsilon_{\infty}}\left(\int_{-\infty}^{+\infty} \frac{\mathrm{d} q_{z}}{q_{z}^{2}+\left|K_{1}-K_{1^{\prime}}\right|^{2}} F_{11^{\prime}}\left(q_{z}\right) F_{22^{\prime}}\left(q_{z}\right)\right) \delta_{K_{2}-K_{2^{\prime}}, K_{1^{\prime}}-K_{1}},
$$


where

$$
F_{i j}\left(q_{z}\right)=\frac{1}{L} \int_{\frac{-L}{2}}^{\frac{ \pm L}{2}} \mathrm{~d} z \exp \left(\mathrm{i} q_{z} z\right) \sum_{\nu^{\prime}} \chi_{\nu_{i} \nu^{\prime}}^{n_{i}^{*}} \chi_{\nu_{j} \nu^{\prime}}^{n_{j}}
$$

is the form factor.

The Coulomb potential has to be screened by the high frequency dielectric constant $\varepsilon_{\infty}$ [8]. $A$ is the in-plane normalisation area and $\Omega=A \cdot L$. Similar formulae like (5) and (6) arise from a many-band envelope theory for a Q1D structure. As far as $\mathrm{PbTe} / \mathrm{PbSnTe}$ is concerned the band offset is small and only a few subbands exist. We assume, that only the lowest subbands contribute to the recombination. The matrix of envelope functions follows from a system of differential equations corresponding to the bulk $\boldsymbol{k} \cdot \boldsymbol{p}$-analysis. However, the boundary conditions for the envelope functions cannot be fulfilled completely. It is possible to overcome this difficulty by using more complicated band structure models [9], but for a first estimate of the effect it is sufficient to use a simple-band approach corresponding to a wave function of the form

$$
\Psi_{\nu}^{n}(\boldsymbol{r})=\exp (\mathrm{i} \boldsymbol{K} R) \chi_{\nu}^{n}(z) u_{\nu} \boldsymbol{K}(\boldsymbol{r}) .
$$

This envelope function does not depend on the in-plane wavevector $K$, whereas the cell periodic Bloch factor $u_{\nu} K^{(r)}$ does. In the expression (6) for the matrix element we have to include the overlap integrals $B_{11}$, between the Bloch factors. According to the bulk theory [10]

$$
\left|B_{11^{\prime}}\right|^{2}=\frac{1}{E_{\mathrm{G}}}\left[p_{x}^{2}\left(k_{x_{1}}-k_{x_{1}}\right)^{2}+p_{y}^{2}\left(k_{y_{1}}-k_{y_{1}}\right)^{2}\right] .
$$

Using (8), an upper estimate of the form factors can be found easily for infinite wells [4]. We finally get a simple result for the matrix element $M_{i f}^{\mathrm{Q} 2 \mathrm{D}}$, but not all integrals in (3) can be done analytically.

Let us consider a Q1D electron gas confined in an infinitely deep square well with electronic states approximated by the simple-band envelope theory

$$
\Psi_{\nu k_{z}}^{n, m}(r)=\frac{1}{\sqrt{\Omega}} \exp \left(\mathrm{i} k_{z} z\right) \chi_{\nu}^{n}(x) \chi_{\nu}^{m}(y) u_{\nu k_{z}}(r)
$$

Under the same assumptions as for the Q2D case we obtain the matrix element

$$
\left|M_{i f}^{1 \mathrm{P}}\right|^{2}=\frac{1}{2}\left(\frac{9}{4}\right)^{2} \frac{\hbar^{2}}{\Omega^{2}}\left(\frac{e^{2}}{\varepsilon_{o} \varepsilon_{\infty}}\right)^{2} \frac{1}{E_{\mathrm{G}} m_{z}} \delta_{k_{z_{1}}-k_{z_{1}}, k_{z_{z^{\prime}}}-k_{z_{2}}} .
$$

For the Q1D model it is possible to take all the integrals in (3) only using the usual approximations [11]. Finally we get 


$$
\begin{array}{r}
C_{A}^{1 \mathrm{D}}=\frac{W^{1 \mathrm{D}}}{\left(1+\frac{3}{4} \frac{k_{\mathrm{B}} T}{E_{\mathrm{G}}^{1 \mathrm{D}}}\right)^{3}} \hbar^{3}\left(\frac{e^{2}}{\varepsilon_{o} \varepsilon_{\infty}}\right)^{2}\left(\frac{3}{2}\right)^{4} \sqrt{\pi} \sqrt{\frac{m_{\perp}}{m_{\beta}}} \frac{1}{m_{\alpha}^{2}} \frac{1}{\left(E_{\mathrm{G}}^{1 \mathrm{D}}\right)^{\frac{5}{2}}} \times \\
\frac{1}{\sqrt{k_{\mathrm{B}} T}} \exp \left(-\frac{E_{\mathrm{a}}^{1 \mathrm{D}}}{k_{\mathrm{B}} T}\right)
\end{array}
$$

where

$$
W^{1 \mathrm{D}}=\mu^{1 \mathrm{D}} /\left(1+\frac{\sqrt{m_{\beta}}+\sqrt{m_{\gamma}}+\sqrt{m_{\delta}}}{\sqrt{m_{\alpha}}}\right)^{3}
$$

denotes multiplicities which are caused by the many-valley structure. The value of $\mu^{1 \mathrm{D}}$ lies between 2 and $4, m_{\alpha}, m_{\beta}, m_{\gamma}, m_{\delta}$ are the effective masses of the four valleys entering the Q1D problem, the masses $m_{\|}, m_{\perp}$ correspond to the main axes of the $L$-valleys in the bulk.

Now we compare the Q1D Auger coefficient (12) with the corresponding bulk expression [10]

$$
\begin{array}{r}
C_{\mathrm{A}}^{3 \mathrm{D}}=\frac{W^{3 \mathrm{D}}}{\left(1+\frac{15}{4} \frac{k_{\mathrm{B}} T}{E_{\mathrm{G}}^{3 \mathrm{D}}}\right)^{3}} \hbar^{3}\left(\frac{e^{2}}{\varepsilon_{o} \varepsilon_{\infty}}\right)^{2} \frac{\sqrt{\pi}}{8} \frac{\left(1+\frac{m_{\perp}}{m_{\|}} \frac{E_{\mathrm{G}}^{3 \mathrm{D}}}{k_{\mathrm{B}} T}\right)}{\sqrt{m_{\|}}\left(\sqrt{m_{\perp}}\right)^{3}} \times \\
\frac{1}{\left(E_{G}^{3 \mathrm{D}}\right)^{\frac{7}{2}}} \exp \left(-\frac{E_{\mathrm{a}}^{3 \mathrm{D}}}{k_{\mathrm{B}} T}\right) .
\end{array}
$$

Here, $W^{3 \mathrm{D}}=(w-1) / w^{2}$ with the number of valleys $w=4$. The ratio $\eta=C_{\Lambda}^{1 \mathrm{D}} / C_{\mathrm{A}}^{3 \mathrm{D}}$ is given by

$$
\begin{array}{r}
\eta=\left[\frac{81}{2} \frac{W^{1 \mathrm{D}}}{W^{3 \mathrm{D}}} \frac{m_{\perp}^{2} \sqrt{m_{\|}}}{m_{\alpha}^{2} \sqrt{m_{\beta}}}\left(\frac{E_{\mathrm{G}}^{3 \mathrm{D}}}{E_{\mathrm{G}}^{1 \mathrm{D}}}\right)^{\frac{5}{2}}\right]\left[\left(\frac{1+\frac{15}{4} \frac{k_{\mathrm{B}} T}{E_{\mathrm{G}}^{3 \mathrm{D}}}}{1+\frac{3}{4} \frac{k_{\mathrm{B}} T}{E_{\mathrm{G}}^{1 \mathrm{D}}}}\right)^{3} \frac{\frac{E_{\mathrm{G}}^{3 \mathrm{D}}}{k_{\mathrm{B}} T}}{\left(1+\frac{m_{\perp}}{m_{\|}} \frac{E_{\mathrm{G}}^{3 \mathrm{D}}}{k_{\mathrm{B}} T}\right)}\right] \times \\
\exp \left(-\frac{E_{\mathrm{G}}^{1 \mathrm{D}}-E_{\mathrm{a}}^{3 \mathrm{D}}}{k_{\mathrm{B}} T}\right) .
\end{array}
$$

Depending on the Q1D direction and the well area the first bracket in (14) changes between 2 and 10 . The second, temperature dependent term, varies from 5 at $200 \mathrm{~K}$ to an asymptotic value of 10 for decreasing temperatures. For $T$ smaller than $77 \mathrm{~K}$ essentially the exponential term decreases the ratio $\eta$. Choosing a direction with high enough Q1D activation energy the Auger recombination rate can be strongly reduced in a Q1D structure compared to the bulk rate. 


\section{References}

[1] Y. Arakawa, A. Yariv, IEEE J. Quantum Electron. 22(9), 1887 (1986).

[2] M. Kriechbaum, P. Kocevar, H. Pascher, G. Bauer, IEEE J. Quantum Electron. 24, 1727 (1988);

S. Shimomura, Y. Urakawa, S. Takaoka, K. Murase, A. Ishida, H. Fujiyasa, Superlattices Microstruct. 7, 5 (1990).

[3] M. Mocker, O. Ziep, Phys. Status Solidi B 115, 415 (1983).

[4] R.I. Taylor, R.A. Abram, M.G. Burt, C. Smith, IEE Proc. 132, 364 (1985).

[5] M. Takeshima, Phys. Rev. B 31, 992 (1985).

[6] M. Mocker, F. Lemke, P. Selbmann, Phys. Status Solidi B 151, K151 (1989).

[7] M. Altarelli, Phys. Rev. B 28, 842 (1983).

[8] O. Ziep, M. Mocker, Phys. Status Solidi B 119, 299 (1983).

[9] M.G. Burt, Semicond. Sci. Technol. 3, 739 (1988).

[10] M. Mocker, O. Ziep, M. Beiler, Wiss. Z. Humboldt-Univ. Berlin, Math.-Natwiss. Reihe 35, 165 (1986).

[11] A.R. Beattie, P.T. Landsberg, Proc. R. Soc. Lond. A, Math. Phys. Sci. 249, 16 (1959). 